\title{
On the influence of the vertical density structure on the dynamics of small basins, with specific application to the Adriatic Sea
}

\author{
P. Malanotte Rizzoli (**) - D. Gazzillo
}

Received on November 15 th, 1976

\begin{abstract}
SUMmary. - The importance of a vertical density structure is discussed for what the hydrologic response and circulation pattern of small basins are concerned. The existence of a density stratification is parameterized through a penetration depth of vertical mixing, and different physical situations are analyzed under a specific set of model assumptions and in basins of sizes similar to the Adriatic Sea.

A specific application is made for the Winter hydrological situation in the Adriatic.

Riassunto. -- Viene discussa limportanza di una struttura verticale della densità nel determinare la risposta idrologica ed il eampo di circolazione in bacini di piccole dimensioni. La stratificazione verticale della densita d parametrizzata tramite una profondita di penetrazione del mescolamento verticale. Situazioni fisiche diverse vengono analizzate in base ad assunzioni specifiche valide per bacini di dimensioni simili al mare Adriatico, la cui situazione idrologica invernale viene analizzata tramite un applicazione particolare del modello ed il confronto coi dati sperimentali.
\end{abstract}

\section{1) Ixtroduction}

In this paper, we analyze the dynamical behaviour of small basins, with geometrical scales similar to those of the Arlriatic Sea,

$\left(^{* *}\right)$ Laboratorio Studio Dinamica Grandi Masse - C.N.R. - S. Polo 1364 Venezia. 
in which a dominant role in determining the hydrographic response and the circulation pattern is played by a complex - and a priori completely arbitrary density structure.

Particular emphasis is given to the importance of the rertical dependence in the density function through the definition of a "penetration repth" $D_{s}$, which is the scale depth of vertical change of the density profile. Different values of the penetration depth $D_{\text {s }}$ determine the relative importance of the natural time scales of the motion, and characterize therefore completely different dynamical behaviours of the considered basin. A discussion is given of the assumptions involved in adimensionalizing and approximating the equations of motion, accolding to the various physical cases defined by different values of the $\omega_{s}$ and in the context of a regular expansion procerlure in a characteristic small parameter. We then analyze in detail the specific case which idealizes a characteristic Winter hychological situation of the $\Lambda$ driatic Sea. This situation has been rescriber and modelled mathematically in a previous paper (Hendershott and Rizzoli) ( ${ }^{1}$. Here we justify through a rigorous procerlure the basic physical assumption of the over-mentioned morlel, deriving again the established fundamental theoretical results which were moreover confirmed by the experimental evidence (Hendershott-Rizzoli; Malanotte Rizzoli) (1,2).

\section{2) TIE Governivg equations}

We consider a rotating, stratified, incompressible, hydrostatic ("thin" system, aspect ratio of the basin $\lambda=\frac{D_{0}}{X} \ll 1$ ) Boussinesq fluid. In basins of the sizes of the Adriatic Sea, the beta effect can be neglecterl; the Coriolis parameter is therefore assumed constant (f-plane) and given by the average latiturle of the considered basin. If, moreover, one makes the assumption that density is linearly related to both temperature and salinity, one can express heat and salt conservation through a single equation for mass conservation.

We follow the traditional approach in ocean circulation modelling of expressing viscous terms through constant edly viscosity and eddy diffusivity coefficients. Even if these coefficients represent a very rough model of the complex dynamics involved in the representation of the viscous stress terms, this classical line of approach can 
still give a very considerable insight in the understanding of the dynamics of circulation phenomena and it is widely used in recent and fundamental fluid dynamies studies (Pedlosky) $(\mathbf{3 , 4 , 5})$.

In these hypotheses, the dimensionful equations of motion are:

$$
\begin{aligned}
& u_{t^{\prime}}^{\prime}+u^{\prime} u_{x^{\prime}}^{\prime}+v^{\prime} u_{y^{\prime}}^{\prime}+w^{\prime} u_{z^{\prime}}^{\prime}-f v^{\prime}=-p^{\prime}{ }_{x^{\prime}} / \varrho_{0}+A_{v} u_{z^{\prime} z^{\prime}}+A_{H} \nabla_{H^{2}} u^{\prime} \\
& v^{\prime}{ }_{t^{\prime}}+u^{\prime} v_{x^{\prime}}^{\prime}+v^{\prime} v_{y^{\prime}}^{\prime}+w^{\prime} v_{z^{\prime}}^{\prime}+f u^{\prime}=-p^{\prime}{ }_{y^{\prime}} / \varrho_{0}+A_{v} v_{z^{\prime} z^{\prime}}+\Lambda_{H} \nabla_{H^{n}} v^{\prime} \\
& O=-p^{\prime}{ }^{\prime} / 0-g \\
& u^{\prime} x^{\prime}+v^{\prime}{ }_{y^{\prime}}+w_{z^{\prime}}^{\prime}=0 \\
& \varrho_{t^{\prime}}+u^{\prime} \varrho_{x^{\prime}}+v^{\prime} \varrho_{y^{\prime}}+v^{\prime} \varrho_{z^{\prime}}=K_{v} \varrho_{z^{\prime} z^{\prime}}+K_{H} \nabla_{H^{2} \varrho}
\end{aligned}
$$

with the usual meaning of symbols. (*)

Here, subscripts indicate partial derivatives, and:

$A_{v}=$ vertical eddy viscosity coefficient

$A_{I}=$ horizontal eddy viscosity coefficient

$K_{v}=$ vertical eddy diffusivity coefficient

$K_{H}=$ horizontal eddy diffusivity coefficient

$\varrho_{0} \quad-$ mean density of the basin

$\nabla_{H}^{2}=\frac{\partial^{2}}{\partial x^{2}}+\frac{\partial^{2}}{\partial y^{2}}=$ horizontal Laplacian

We proceed therefore to adimensionalize the equations through the following scales:

$$
\begin{aligned}
\left(x^{\prime}, y^{\prime}\right) & =\boldsymbol{X}(x, y) \\
z^{\prime} & =D_{\mathrm{o}} z
\end{aligned}
$$

with $-d \leqslant z \leqslant 0$ and $d=\operatorname{depth} / D_{0}$ is the dimensionless variable depth;

Coriolis parameter;

$$
f=f_{0} \Phi \equiv f_{0}
$$

$$
\varrho=o_{0}+10^{-3} \sigma=\varrho_{0}+10^{-3} \Delta \sigma \cdot S
$$

where $\sigma$ is nearly the usual $\sigma_{t}$ (essentially $\sigma=\sigma$, in seas as shallow as the Northern Adriatic), $\Delta \sigma$ is its observed maximum spatial variation and $S$ is the dimensionless density;

$$
\left(u^{\prime}, v^{\prime}\right)=U(u, v)
$$

(*) For completeness, a table of all symbols can be found at the end. 
with

$$
C=g D_{0} 10^{-3} \Delta \sigma / f_{0} X \varrho_{0}
$$

as given by thermal wind relationship;

$$
p^{\prime}=\bar{p} p
$$

after subtraction of the hydrostatic pressure due to the mean density Qo, according to the usual convention, and

$$
\bar{p}=\varrho_{0} 10^{-3} g \Delta \sigma D_{0} .
$$

The chosen scale for horizontal velocities (that is given by thermal wind relationship) plus a realistic choice for the eddy coefficients in the range of ralues suitable for basins of sizes comparable to the Adriatic Sea, imply that we deal with circulation models characterized by a geostrophic interior and two, top and bottom, Ekman layers. As in general deep sea circulation models, these Ekman layers will be used in the context of boundary layer approach to satisfy top and bottom boundary conditions for the velocity components; they are, therefore, thin in respect to the total depth.

As a consequence, it can be shown that the vertical velocity $w^{\prime}$ has a scale depth of vertical change given by the Ekman depth:

$$
\begin{aligned}
& D_{e}=\sqrt{A_{v} / f_{0}} \\
& w^{\prime}=W w
\end{aligned}
$$

with

$$
W=\sqrt{A_{v} / f_{0} I_{0}^{2}} \cdot I_{0} U / X
$$

as set by Ekman pumping.

In the case of the Adriatic Sea, the previous scales have the following values:

$$
\begin{aligned}
X & =100-200 \mathrm{Km} . \\
D_{0} & =100 \mathrm{~m} \\
f_{0} & =10^{-1} \mathrm{sec}^{-1} \\
\Delta \sigma & \simeq 0.5 \mathrm{gr} / \mathrm{cm}^{3} \\
E & \simeq 5 \mathrm{~cm} / \mathrm{sec} \\
A_{v}-K_{0} & -10^{2} \mathrm{~cm}^{2} / \mathrm{sec} \\
A_{1}-K_{I I} & =10^{6} \mathrm{~cm}^{2} / \mathrm{sec} \\
D_{e} & =10^{\mathrm{m}}
\end{aligned}
$$

For what the time scale of the motion is concerned

$$
t^{\prime}=T t
$$


we can define three natural time scales of the system:

$$
\begin{aligned}
T_{a l v} & =X / U \text { advective time scale } \\
T_{v d} & =D_{0}^{2} / K_{v} \text { vertical diffusion time scale } \\
T_{I I d} & =\Gamma-/ K_{n} \text { horizontal diffusion time scale }
\end{aligned}
$$

According to the dominant physical mechanism, the time scale will be defined to be [2] (a); (b); (c) respectively.

With the choice of these scales, the adimensionalized equations of motion become:

$$
\begin{aligned}
\varepsilon_{T} u_{\iota}+\varepsilon_{R}\left(u u_{x}+v u_{y}+\varepsilon_{v}^{1 / 2} w u_{z}\right)-v & =-p_{x}+\varepsilon_{v} u_{z z}+\varepsilon_{I I} \nabla_{u^{2}} u \\
\varepsilon_{T} v_{\iota}+\varepsilon_{R}\left(u v_{x}+v v_{y}+\varepsilon_{v}^{1 / 2} w v_{z}\right)+u & =-p_{y}+\varepsilon_{v} v_{z z}+\varepsilon_{I I} \nabla_{I^{2}} v \\
O & =-p_{z}-S \\
u_{x}+v_{y}+\varepsilon_{v}^{1 / 2} w_{z} & =0 \\
\Gamma_{T} S_{\iota}+u S_{x}+v S_{y}+\varepsilon_{v}^{1 / 2} w S_{z} & =\Gamma_{v} S_{z z}+\Gamma_{I I} \nabla_{I I} S^{\prime}
\end{aligned}
$$

where

$$
\nabla_{H^{2}}=\partial^{2} / \partial x^{2}+\partial 2 / \partial y^{2}
$$

and the dimensionless parameters are:

$$
\varepsilon_{T^{\prime}}=1 / f_{0} T^{\prime}
$$

with the already mentioned three possible choices for $T$

$$
\varepsilon_{n}=U / f_{0} X
$$

Rossby number $\left(\varepsilon_{n}=\varepsilon_{T}\right.$ if the time scale is the adrective one)

$$
\varepsilon_{v}-A_{v} / f_{0} D_{0}=
$$

vertical Ekman number

$$
\varepsilon_{n}=A_{H} / f_{0} X^{2}
$$

Horizontal Ekman number

$$
\begin{aligned}
& I_{T}-T_{\text {adr }} / T \\
& T_{v}=T_{\mathrm{ad} d} / T_{\mathrm{rd}} \\
& \Gamma_{\|}-T_{\text {adr }} / T_{I I d} \\
& \Gamma_{T}=1 ; \Gamma_{T}=-\Gamma_{v} ; \Gamma_{T}=\Gamma_{H} \text { according to the choice for } T \text {. }
\end{aligned}
$$

2.1) The penetration depth and the natural time seales of the motion.

To have a suitable parameter directly related to the rertical structure of the density field, we define a scale depth $I_{s}$ of rertical 
change of the density profile by supposing horizontal advection and vertical diffusion to be of the same order:

$$
I_{s}{ }^{2}=\Pi_{v} \mathrm{X} / \mathrm{U}
$$

is therefore the depth of penetration of rertical mixing. With this parametrization, we can express:

$$
\begin{aligned}
& \Gamma_{v}=T_{a d v} / T_{v d}=D_{s^{2}} / D_{0^{2}} \\
& \Gamma_{H}=T_{a d v} / T_{I d}=\Pi_{H} / \mathrm{X} U=K_{H} D_{s^{2}} /\left(K_{r} \mathrm{I}^{2}\right)
\end{aligned}
$$

With these rlefinitions, it is intuitive to see how the seale of penetration of rertical mixing $D_{s}$ is related to the time scale of the motion and, therefore, to the dominating physical mechanism. If $\Gamma_{v} \ll 1$, this means $D_{s} \ll D_{0}$ and $T_{a d r} \ll T_{v d}$; the rertical mixing will be limited to a smaller depth as compared to the total one, that is the time necessary for the vertical diffusive process to produce vertical homogeneity in the density field will be much longer than the time required by a water column to make a complete tour in the circulation gyre. If in this case we choose as time scale the smaller $T_{\text {add }}$, we'll describe the time evolution of the density field as determined primarily by adrection. The clensity field, on the other sicle, will be stationary (to order zero) when time will have elapsed as to reach the order of magniturle of the $T_{r d}$ scale.

The opposite situation happens if $\Gamma_{v} \gg 1$, that is $D_{s} \gg D_{0}$ or $T_{a d v} \gg T_{v d}$. The time necessary for vertical mixing to be complete is now much smaller than the arvection time. We'll choose the $I_{v d}$ time scale if we want to describe the details of the time evolution of the vertical mixing process in the density field. If, on the other hand, we limit ourselves to consider motions with time scales of the orcler of $T_{\text {adr }}$ or larger, these motions will see the vertical distribution of the density field as completely homogeneous, and for them the vertical diffusion process will be essentially instantaneous $\left(T_{v d}=0\right)$.

The time scale $T_{I n}$ is the greatest of the three. In the limit of time seales of this order, it can be shown, in the context of the expansion procelure we are exposing in the following, that the density field $S$ has reached the stationary situation, as well as the velocity fielıl. In fact:

$$
\begin{aligned}
T_{a d v} & =\Gamma_{H} T_{H d} \\
T_{v d} & =\frac{\Gamma_{H}}{\Gamma_{v}} T_{H d}
\end{aligned}
$$


Being $\Gamma_{\|}$a small dimensionless parameter (in the case of the Ariatic $\left.\Gamma_{I I} \simeq 2 \cdot 10^{-2}\right), T_{a d v} \ll T_{I d d}$. If $\Gamma_{v} \gg 1$, then $T_{v d l} \ll T_{I I d}$. In the opposing limit $\Gamma_{v} \ll 1, T_{v d}$ and $T_{n d}$ can become of the same orcler of magnitude, and the choice of either time scale is equiralent to describe the physical process. We therefore limit ourselves to consider the two time scales $T_{a d v}$ and $T_{r d}$ in the two extreme cases $\Gamma_{v} \ll 1$ and $\Gamma_{v} \gg 1$.

To further specify the physical situation we'll cleal with, we make some assumptions on the relative magnitude of the climensionless small parameters appearing in the momentum equation. For what the time dependence is concerned

$$
\begin{array}{ll}
\varepsilon_{T}=1 / f_{\mathrm{o}} T=\varepsilon_{R} & \text { if } T=T_{\mathrm{ad} v} \\
\varepsilon_{T}=\Gamma_{v} \varepsilon_{R} & \text { if } T=T_{\mathrm{v} d}
\end{array}
$$

Therefore, even with a very small Rossby number, in the case of motions with time scale $T_{v d}$ and $\Gamma_{v} \gg 1$, the time dependence in the momentum equations might not be negligible.

We put ourselves in the following limit, which is valid for basins similar in size to the Arriatic Sea:

$$
\begin{aligned}
& \varepsilon_{H}<\varepsilon_{H}<\varepsilon_{v} \ll 1 \\
& \Gamma_{H} \sim 0\left(\varepsilon_{v}\right)
\end{aligned}
$$

With the scales suitable for the Arlriatice Sea, in fact,

$$
\begin{array}{ll}
\varepsilon_{H}=A_{H} / f_{0} X^{-2} \simeq 10^{-4} & \varepsilon_{v}=A_{v} / f_{0} D_{0}^{2} \simeq 10^{-2} \\
\varepsilon_{R}=U / f_{0} X \simeq 5 \cdot 10^{-3} & \Gamma_{H} \simeq 2 \cdot 10^{-2}
\end{array}
$$

In the momentum equations we therefore neglect the non linear advective terms and the lateral diffusion terms in respect to rertical diffusion terms, keeping for the moment the time dependence which might become important in one specific case, as previously mentioned. Our morlel equations will be:

$$
\begin{aligned}
\varepsilon_{T} u_{t}-v & =-p_{x}+\varepsilon_{v} u_{z z} \\
\varepsilon_{T} u_{t}+u & =-p_{y}+\varepsilon_{v} v_{z z} \\
O & =-p_{z}-S \\
u_{x}+v_{y}+\varepsilon_{v}{ }^{1 / 2} w_{z} & =0 \\
J_{T} S_{1}+u_{x}+S_{y}+\varepsilon_{v}{ }^{1 / 2} w S_{z} & =\Gamma_{v} S_{z z}+\Gamma_{u} \nabla_{u}{ }^{2} S
\end{aligned}
$$


with:

$\Gamma_{T}=1 ; \varepsilon_{T}=\varepsilon_{R}$ (negligible time depentlence) if $T=T_{a d v}$

$\Gamma_{T}=\Gamma_{v} ; \varepsilon_{T}=\Gamma_{v} \varepsilon_{R}$

if $T=T_{v t l}$.

3) THE EXPANSION PROCEDURE AND THE DIFFERENT LIMITING CASES

We choose $I_{H}$ as the small dimensionless parameter characteristic of the system and we take a power of $\Gamma_{H}$, let us say $\Gamma_{I I}^{a}$, as the small parameter of a regular perturbation expansion of all the field functions:

$$
\left(\begin{array}{l}
u \\
v \\
w \\
p \\
S
\end{array}\right)=\sum_{n=o}^{\infty}\left(\Gamma_{H}^{\alpha}\right)^{n}\left(\begin{array}{c}
u^{(n)} \\
v^{(n)} \\
w^{(n)} \\
p^{(n)} \\
S^{(n)}
\end{array}\right)
$$

In principle, the value of $\alpha$ is completely general. Even without specifying it, we restrict ourselves to the following hypothesis for what oriters of magnitude are concernesl

$$
I_{i t}^{a} \sim 0\left(\varepsilon_{v}^{1 / 2}\right)
$$

which implies $0<a \leqslant 1$ for the basins we are considlering, similar in size to the Arlriatic Sea.

Therefore, to every order in $\Gamma_{M}^{u}$, the velocity field can be split into a geostrophic interior plus two top and bottom Ekman layers. The details of Ekman layer analysis will be given in paragraph (4). With this in mind, let us consisler separately the two chosen natural time scales and, in the context of each of them, the two limiting cases $\Gamma_{v} \ll 1$ and $\Gamma_{v} \gg 1$.

3.1) $T=T_{r d}$

The morlel equations are:

$$
\begin{aligned}
& \Gamma_{r} \varepsilon_{l} u_{\ell}-v=-p_{x}+\varepsilon_{v} u_{z z} \\
& \Gamma_{v} \varepsilon_{R} v_{\imath}+u=-p_{u}+\varepsilon_{v} v_{z z} \\
& O=-p_{z}-S \\
& u_{x}+v_{y}+\varepsilon_{v^{1 / 2}} w_{z}=0 \\
& \Gamma_{u} S_{t}+w S_{x}+v S_{y}+\varepsilon_{v}^{1 / 2} u S_{z}=\Gamma_{t} S_{z z}+\Gamma_{u} \nabla_{u}-S
\end{aligned}
$$


Many subcases might be distinguisherl according to the relative magnitude of $\Gamma_{v}$ and $\Gamma_{I I}^{\alpha}$. We'll limit ourselves, anyway, to the most significant ones, always in the context of expansion [7].

3.1a) $\Gamma_{v} \ll 1$

Oriter zero

$$
\begin{aligned}
-v^{(0)} & =-p_{x}(0)+\varepsilon_{v} u_{z z}(0) \\
u^{(0)} & =-p_{y}{ }^{(0)}+\varepsilon_{v} v_{z z}(0) \\
S^{(0)} & =-p_{z}{ }^{(0)} \\
u_{x}(0)+v_{y}(0)+\varepsilon_{v}{ }^{1 / 2}\left(u_{z}^{(0)}\right. & =0 \\
u^{(0)} S_{x}(0)+v^{(0)} S_{y}(0) & =0
\end{aligned}
$$

Order $\Gamma_{H}^{\alpha}$

$$
\begin{aligned}
& -v^{(1)}=-p_{x}^{(1)}+\varepsilon_{v} u_{z z^{(1)}} \\
& u^{(1)}=-p_{y^{(1)}}+\varepsilon_{v} v_{z z^{(1)}} \\
& S^{(1)}=-p_{z}^{(1)} \\
& u_{x^{(1)}}+v_{y}{ }^{(1)}+\varepsilon_{v}{ }^{1 / 2} u_{z}^{(1)}=0 \\
& u^{(0)} S_{x}^{(1)}+v^{(0)} S_{!}^{(1)}+u^{(1) S_{x}^{(0)}}+v^{(1), S_{y}^{(0)}}+\delta w^{(0)} S_{z}^{(0)}=0 \\
& \text { if } \Gamma_{v}<\Gamma_{H}^{\prime a} \\
& v S_{t^{(0)}}+u^{(0)} S_{x^{(1)}}+v^{(0)} S_{y^{(1)}}+u^{(1)} S_{x^{(0)}}+v^{(1)} S_{y^{(0)}}+\delta w^{(0)} S_{z^{(0)}}=\nu S_{z z^{(0)}} \\
& \text { if } I_{v} \sim \Gamma_{i}^{\top u}
\end{aligned}
$$

with $\delta=\varepsilon_{v^{1 / 2}} \Gamma_{H^{-\alpha}} \simeq 0(1)$ and $\gamma=\Gamma_{v} \cdot \Gamma_{. I}^{\alpha} \simeq 0(1)$ according to [8]. In the horizontal momentum plus continuity equations we have hept the $z$-partial derivatives, weighted by $\varepsilon_{1}$, to mean that, to both orders, the total relocity field must be split into a geostrophic interior plus two Ekman contributions which decay outside the two Ekman layers. If, in fact, we should consider the Eliman layer equations, we should first stretch the rertical coordinate in system [9] putting: $z=\varepsilon_{v}^{-1 / 2} z$ or $\bar{z}=\varepsilon_{v}^{1 / 2}(z+d)$ for the top and bottom Ekman layers respectively, and, in the boundary momentum equations, apply the formal expansion procedure in $I_{:}^{\prime \prime}$. The density equations, on the other hand, are the zero and $I_{\| I}^{u}$ orders of the expansion of the geostrophic interior density equations. In this case, as discussed in (2-1), $D_{s} \ll D_{0}$, and the proper time scale to see any time erolution of the density field to zero orler ought to be the smaller $T_{\text {adr. }} \Delta \mathrm{s} T=T_{\text {rdd }}$, and rertical diffusive processes have a longer time scale, the zero order field $S^{(0)}$ is stationary and is a function of a zero order stream function $\psi^{(0)}$ for the relocity, which can be introduced through the continuity equation of the geostrophic interior $\left(u_{x^{(0)}}+v_{y^{(0)}}=0\right)$ that is: $S^{(0)}=f\left(\psi^{(0)}\right)$. 
3.1b) $1 \ll \Gamma_{v}<\varepsilon_{R}^{-1}$

Orcler zero

$$
\begin{aligned}
-v^{(0)} & =-p_{x}(0)+\varepsilon_{v} u_{z z^{(0)}} \\
u^{(0)} & =-p_{y^{(0)}}+\varepsilon_{v} v_{z z}(0) \\
S^{(0)} & =-p_{z^{(0)}} \\
u_{x^{(0)}}+v_{y^{(0)}}+\varepsilon_{v}^{1 / 2} v_{z^{(0)}} & =0 \\
S_{t^{(0)}} & =S_{z z^{(0)}} \\
\text { and } \gamma & =\Gamma_{v} \cdot \Gamma_{I I}^{a} \sim 0(1)
\end{aligned}
$$

Order $\Gamma_{H} a$

$$
\begin{aligned}
& -v^{(1)}=-p_{x^{(1)}}+\varepsilon_{v} u_{z z}{ }^{(1)} \\
& u^{(1)}=-p_{y}^{(1)}+\varepsilon_{v} v_{z z^{(1)}} \\
& S^{(1)}=-p_{z}^{(1)} \\
& u_{x}^{(1)}+v_{y}^{(1)}+\varepsilon_{v}^{1 / 2} w_{z}^{(1)}=0 \\
& \gamma S^{(1)}+u^{(0)} S_{x^{(0)}}+v^{(0)} S_{y^{(0)}}=\gamma S_{z z^{(1)}} \\
& \text { if } \Gamma_{v} \sim \Gamma_{I I}^{-\alpha}
\end{aligned}
$$

In this case, we are in the situation in which rertical mixing is a rery rapicl process compared to horizontal advection, and we are describing its time evolution. The zero order density field $S(0)$ obeys the heat equation in the vertical direction, the solutions of which are well linown, and should be chosen according to the specific boundary conditions to be applied to $S$ at $z=0 ;-d$.

3.1.c) $\Gamma_{v} \gg 1$ and $\Gamma_{v} \geqslant \varepsilon_{R}^{-1}$

This is the only case in which the time dependence in the momentum equations becomes important. The velocity field is not stearly any more, and wave behaviours may well be expected to dominate. The equations for $S^{(0)}, S^{(1)}$ remain identical to case (3.1b).

3.2) $I^{\prime}=T_{a i v}$

The model equations are:

$$
\begin{aligned}
-v & =-p_{x}+\varepsilon_{v} u_{z z} \\
u & =-p_{y}+\varepsilon_{v} v_{z z} \\
O & =-p_{z}-\$ \\
u_{x}+v_{y}+\varepsilon_{v}{ }^{1 / 2} w_{z} & =0 \\
S_{t}+u S_{x}+v S_{y}+\varepsilon_{v}{ }^{1 / 2} w S_{z} & =\Gamma_{v} S_{z z}+\Gamma_{I} \nabla_{H}{ }^{2} S
\end{aligned}
$$

Again, in the context of expansion [7], let us consider the two extreme cases of a very small or a very big depth of penetration of vertical mixing. 
$3 \cdot 2$ a) $\Gamma_{v} \ll 1$

Zero order

$$
\begin{aligned}
-v^{(0)} & =-p_{x}^{(0)}+\varepsilon_{v} u_{z z_{z}}(0) \\
u^{(0)} & =-p_{y}(0)+\varepsilon_{v} v_{z z^{(0)}} \\
S^{(0)} & =-p_{z}(0) \\
u_{x}(0)+v_{y}(0)+\varepsilon_{v} v^{1 / 2} w_{z}^{(0)} & =0 \\
S_{t^{(0)}+u^{(0)} S_{x}(0)}+v^{(0)} S_{y}(0) & =0
\end{aligned}
$$

Order $\Gamma_{H} \alpha$

and $v=\Gamma_{v} \cdot I_{H}^{\prime \prime} \sim 0(1)$

$$
\begin{aligned}
& -v^{(1)}=-p_{x^{(1)}}+\varepsilon_{v} u_{z z^{(1)}} \\
& u^{(1)}=-p_{y^{(1)}}+\varepsilon_{v} v_{z z^{(1)}} \\
& S^{(1)}=-p_{z}^{(1)} \\
& u_{x^{(1)}}+v_{y^{\prime}}^{(1)}+\varepsilon_{v} v^{1 / 2} v_{z}{ }^{(1)}=0
\end{aligned}
$$

$S_{i}^{(1)}+u^{(0) S_{x}(1)}+v^{(0)} S_{y^{(1)}}+u^{(1)} S_{x^{(0)}}+v^{(1)} S_{y}^{(0)}+\delta u^{(0)} S_{z}(0)=\left\{\begin{array}{l}0 \\ y S_{z z}(0)\end{array}\right.$

$$
\text { if } \Gamma_{v}^{\top} \ll I_{\underline{u}}^{a} \quad \text { if } \Gamma_{v} \sim \Gamma_{H}^{a}
$$

Again $\delta=\varepsilon_{v}^{1 / 2} \Gamma_{H}^{-\alpha} \sim 0(1)$ according to [8]. Being $\Gamma_{v} \ll 1$, as previously noticed, vertical diffusion is slow compared to horizontal advection. With a time scale characteristic of this latter process, the time evolution of the density field to zero order is determined only by advection.

\section{$3-2 \mathrm{~b}) \Gamma_{v} \gg 1$}

Among the various possible cases, we restrict ourselves to consider only the one $\Gamma_{v} \sim \Gamma_{A}^{-a}$ as the most significant.

Order zero

$$
\begin{aligned}
-v^{(0)} & =-p_{x}(0)+\varepsilon_{v} u_{z z^{(0)}} \\
u^{(0)} & =-p_{y}(0)+\varepsilon_{v} v_{z z^{(0)}} \\
S^{(0)} & =-p_{z}^{(0)} \\
u_{x}(0)+v_{y}(0)+\varepsilon_{v}{ }^{1 / 2} w_{z}^{(0)} & =0 \\
S_{z z^{(0)}} & =0
\end{aligned}
$$

Order $\Gamma_{H}^{\alpha}$

$$
\begin{aligned}
& -v^{(1)}=-p_{x}^{(1)}+\varepsilon_{v} u_{z z^{(1)}} \\
& u^{(1)}=-p_{y^{(1)}}+\varepsilon_{v} v_{z z^{(1)}} \\
& S^{(1)}=-p_{2}^{(1)} \\
& u_{x}^{(1)}+v_{y}^{(1)}+\varepsilon_{v}^{1 / 2} w_{z}^{(1)}=0 \\
& S^{(0)}+u^{(0)} S_{x}^{(0)}+v^{(0)} S_{y}^{(0)}=\gamma S_{z z^{(1)}}
\end{aligned}
$$


In this case, we consider motions with time scales of the order of $T_{a d v}$ or larger; for these motions, the rertical diffusion is essentially instantaneous.

4) The wister pHexomexology of the ADritide SEA AND THE RELATIVE MATHEMATICAL MODEL,

In the previous paragraph we have examined the detailed form of the equations of motion for each of the intrinsic time scales $T_{\text {adv }}$ and $T_{v d}$, and in the extreme, opposite cases of a very small or a very big penetration depth $D_{s}$. We have not tried to give an analytic solution to each specific case - even when the form of the equations allowed it - because every system must be riewed in the context of the physical problem under consicleration, with the suitable and properly chosen boundary conditions. In this paragraph we are concerned with the Winter hydrological situation in the Adriatic sea, discussed and modelled in the previously mentioned papers (see references).

For basins of the shape and dimensions of the Arlriatic, the proper choice and values of the geometrical length scales and of the rarious parameters has been already pointed out in paragraph (2).

In consiclering now specifically the Winter situation, we'll notice that, in most of the Winter hidrological data at disposal, the fields of temperature, salinity, and hence density, are rertically mixed to essential homogeneity. This is, in particular, the case of the two Winter oceanographic campaigns the experimental results of which we'll compare with the theoretical predictions, that is the period January-February 1966 (Trotti 1970) $\left(^{\circ}\right)$ and January-February 1972 (P. Malanotte Rizzoli, in press) (²).

In both these periods, the vertical maps of temperature, salinity, density show an essentially complete rertical mixing, which, in the Southern part of the basin, extends to more than $200 \mathrm{~m}$. (lepth (Fig. 1). Only a narrow boundary strip adjacent to the italian coastline is excluded from this situation of vertical homogeneity. In it, the river outllow - concentrated along the North-Western Italian shoreline causes the persistence of a noticeable vertical stratification persisting also in Winter time.

In the two overmentioned Winters, moreover, there is the formation of a pool of water of particularly high density $(\sigma,>29.4)$ 
ON TIL INFLUECE OF THE VERTICAL DESSITY STRUCTURE ETC. 259

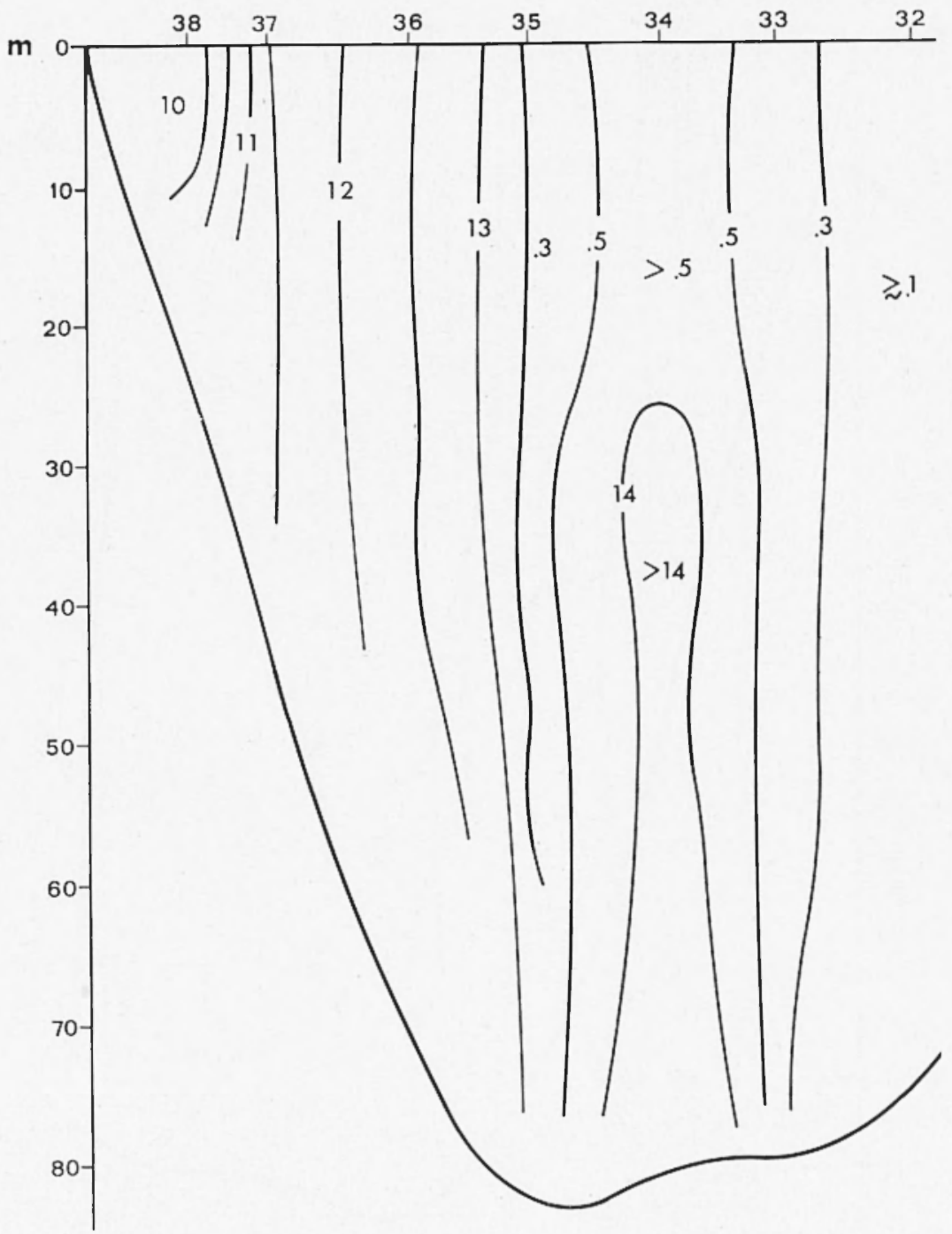

a) Vertical distribution of temperature in oc:

Fig. 1 a-b-c - P. Malanote Rizzoli - Cruise January-February 1972 Vertical maps relative to a cross-section of the Adriatic from Porto Civitanova to the Isola Grossal 


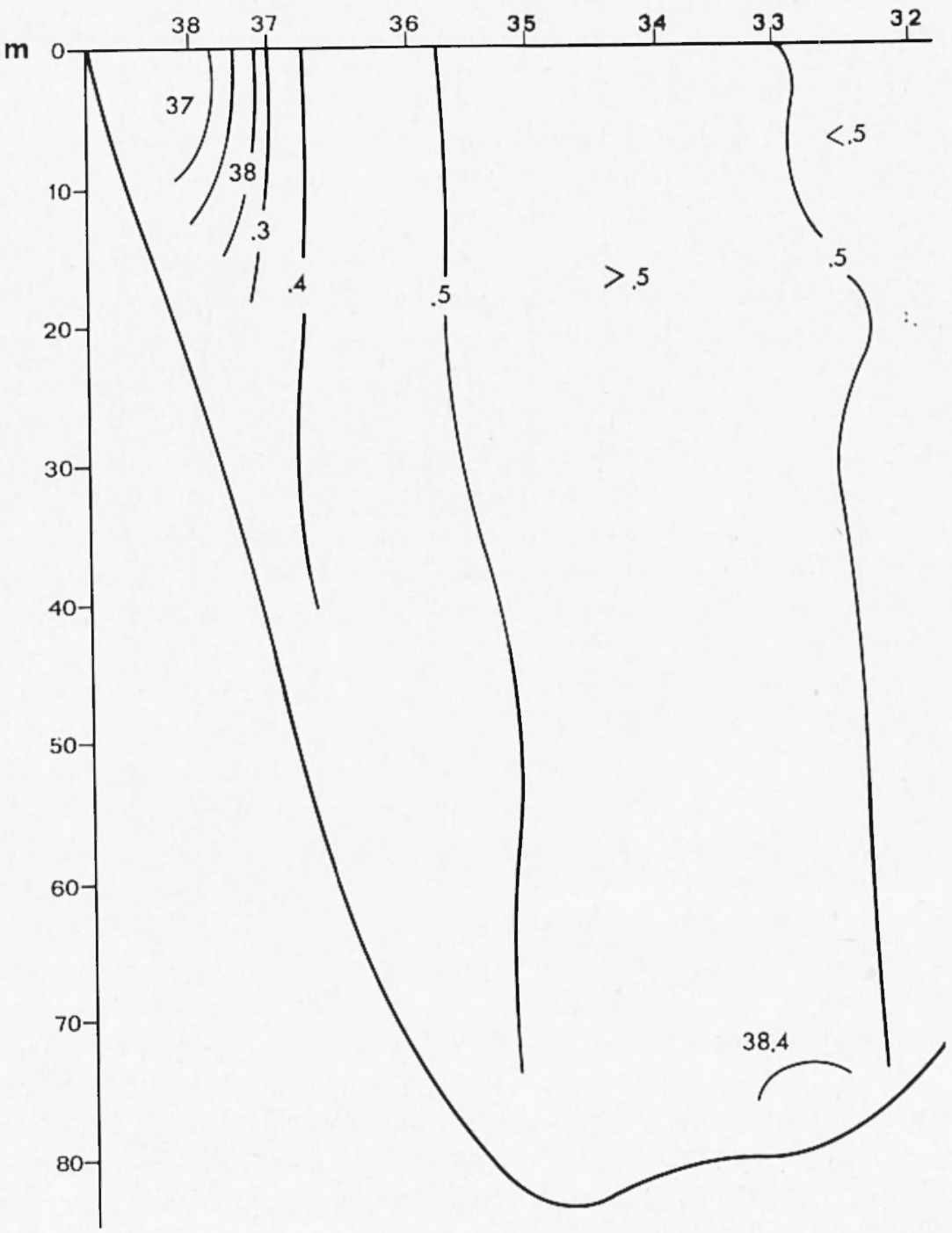

b) Vertical distribution of salinity in ?o. 
ON THE INFluexce OF THE VERTICAL DENeITy strvioture ETC. 261

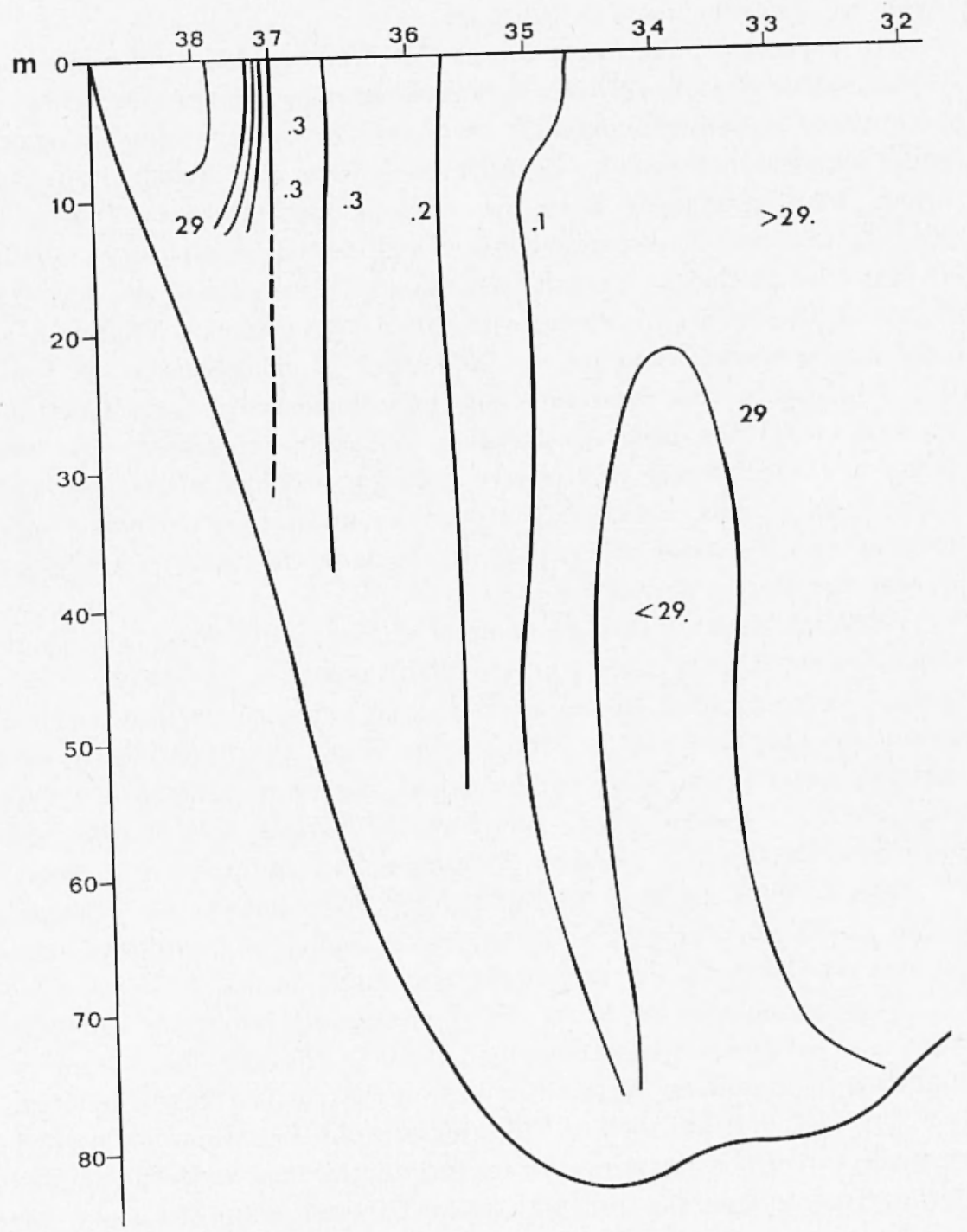

c) Vertical distribution of density anomaly $\sigma_{t}$ 
in the northernmost part of the basin (Fig. 2). This pool protrucles southward with a characteristic "tail" along the western side of the Arliatic, following isobath contours.

In both cases, the formation of this rlense pool can be connected (Hendershott-Rizzoli, 1976 ; P. Malanotte Rizzoli, in press) (1.2) with two exceptional episorles, anomalous in respect to average meteorological conditions, characterized by the outbreak of dry, cold air of continental origin, blowing directly onto the Arriatic from Euroasiatic NorthEastern regions. These episonles - which lasted in both cases about 15 days - produced exceptionally intense thermal and evaporative fluxes at the air-sea interface, with, as a consequence, complete vertical mixing of water columns and the formation of the dense water pool.

To describe and reproduce this hydrological situation, a mathematical molel has been constructer, vertically integrater over the water depth, exploring the relative importance which air-sea thermal fluxes, wind stress, coastal river outflows and water exchange with the Sonthern Adriatic do have in determining the lields of transport stream function $\psi$ and density $S$.

Without entering into the details of the morlel, given elsewhere (Hendershott-Rizzoli, 1976)(1) we'll point out that its primary idealization, corresponding to the experimental evidence, is that vertical mixing of heat and salt is complete for what the dynamies of circulation and the evolution of the density field are concemed. This fundamental hypothesis led, therefore, to assume the density field depending essentially only upon the horizontal coordinates $s=S(x, y)$.

The dependance of $S$ on the vertical coorlinate $z$ was assumed to be exprinible through additive terms negligible, in order of magnitude, relatively to the horizontal, dominant term.

This dependance of $S$ on $z$ was considered important only for what air-sea fluxes were concerned, that is through the boundary condition at the air-sea interface $z=0$ on the vertical flux of density.

This assumption allowed to integrate the equations of motion vertically, over the water depth, lealing to the final model equations in the two functions, the transport stream function $\psi$ and the density $S$.

No rigorous justification was, anyway, given for this basic hypothesis, and the purpose of the following treatment is, as previously mentioned, to prove its validity, deriving it through a formal analytical procedure.

In the context of the development made in this paper, which through the expansion [i] has led to distinguish the various cases 
ON TIE INFLUENCE OF THE VERTICAL Dessity structure etc. 263

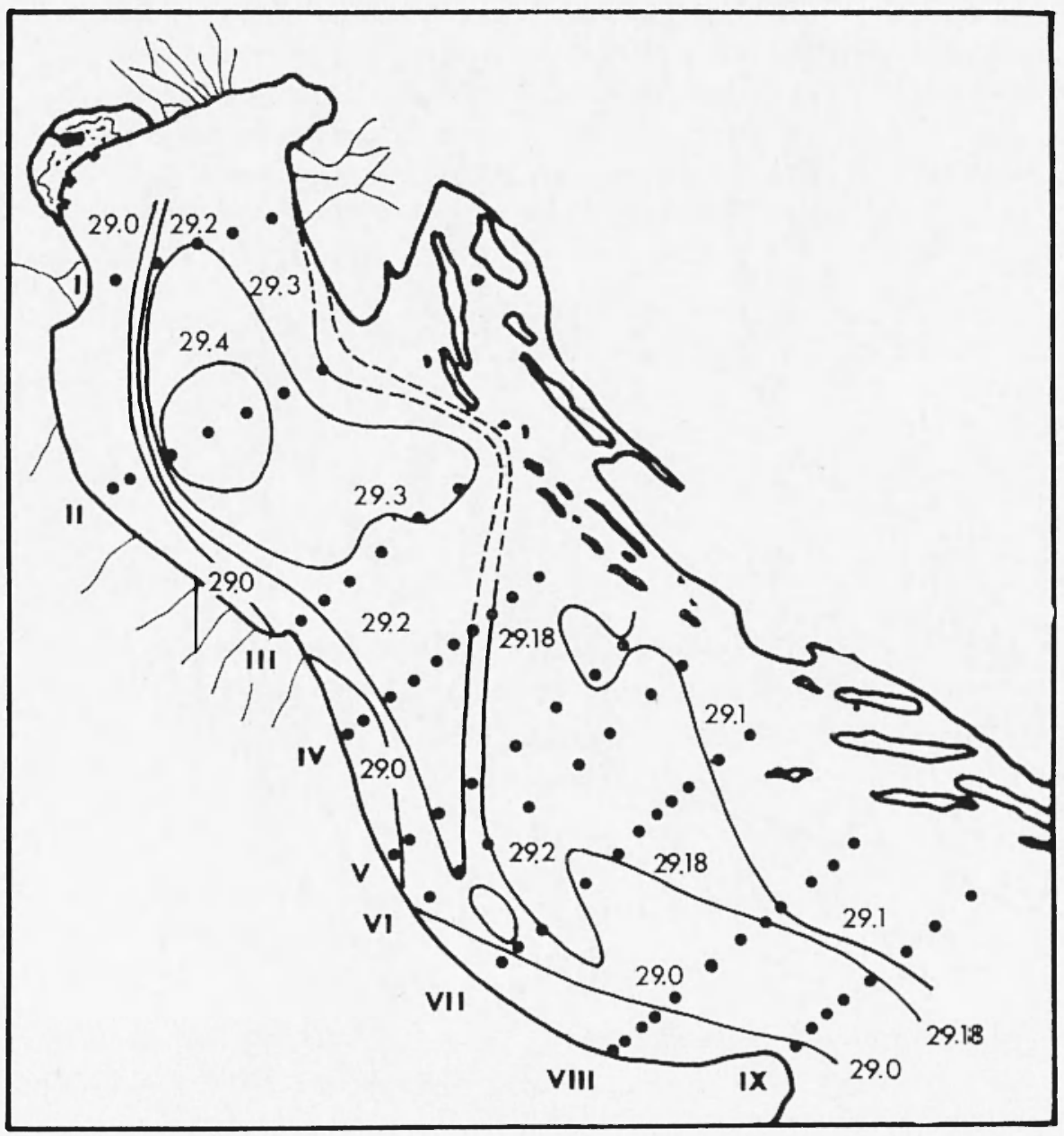

(a) Surface distribution of density anomaly for the cruise January-February 1966

Fig. 2 a-b 


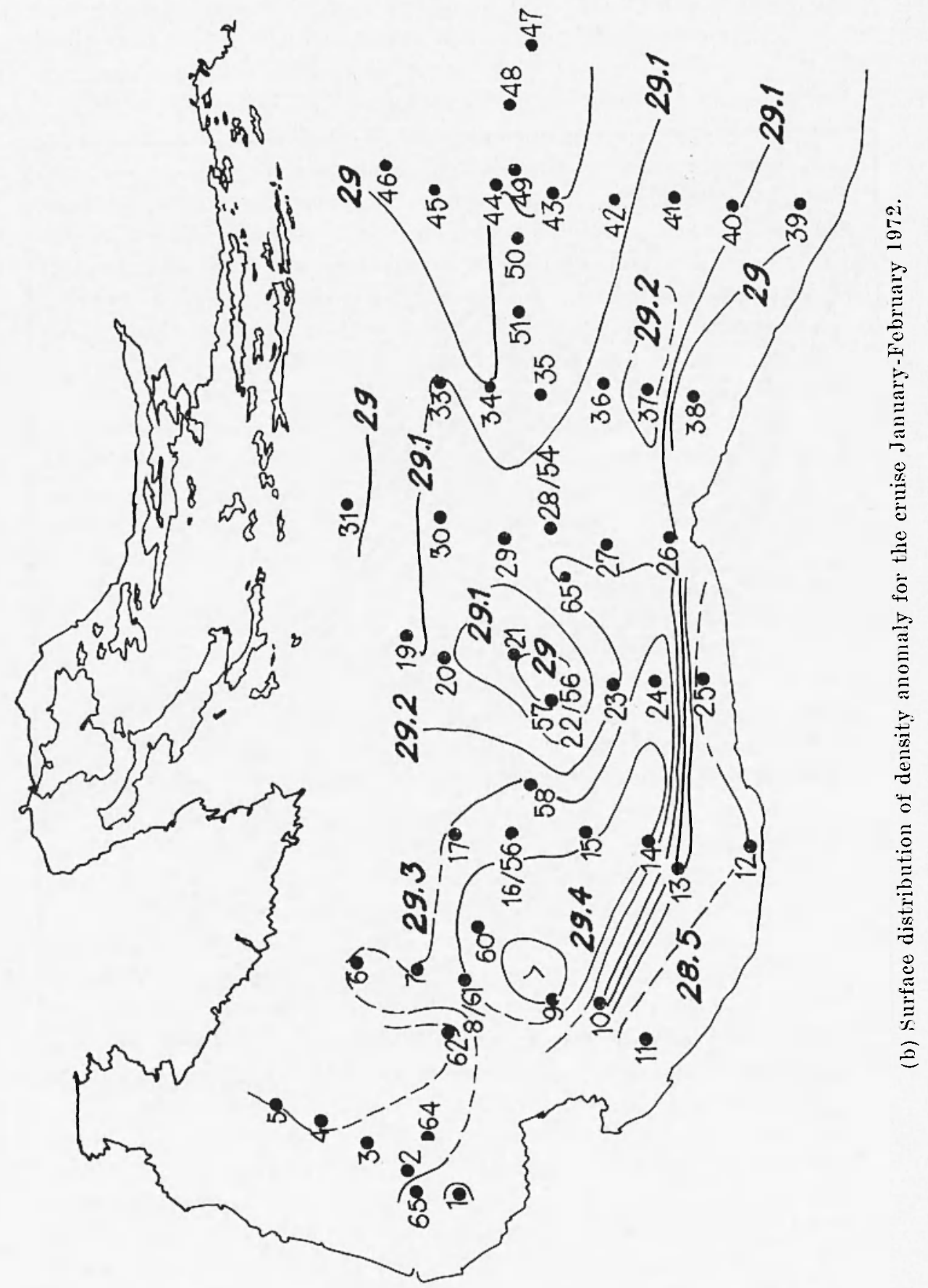


examined in paragraph (3), we are in the situation in which $T_{r d} \ll T_{a d v}$ and $\Gamma_{v} \gg 1$.

The vertical diffusion time scale is very small; vertical mixing can be considered instantaneous $\left(T_{v d} \sim 0\right)$ when dealing with phenomena in which order of magnitude variations in the circulation and density fields occur over times of the order or larger than the advective time scale. We are therefore in the case $[3,2 \mathrm{~b}]$ described by the set of equations [14].

To determine completely the solution to zero order, it is sufficient to couple the zero order system with the order $\Gamma_{J}^{\alpha}$ density equation, getting:

$$
\begin{aligned}
-v^{(0)} & =-p_{x}(0)+\varepsilon_{v} u_{z z}(0) \\
u^{(0)} & =-p_{y}{ }^{(0)}+\varepsilon_{v} v_{z z}(0) \\
S^{(0)} & =-p_{z}(0) \\
u_{x^{(0)}}+v_{y}(0)+\varepsilon_{v}{ }^{1 / 2} u_{z}(0) & =0 \\
S_{z z^{(0)}} & =0
\end{aligned}
$$

and

$$
S_{\iota}^{(0)}+u^{(0)} S_{x^{(0)}}+v^{(0)} S_{y^{(0)}}^{\prime}=\gamma S_{z z^{(1)}}
$$

As previously pointed out, this notation means that two Ekman layers are coupled to a geostrophic interior. In fact, applying the expansion [7] to the interior general equations, the zero order system is:

$$
\begin{aligned}
v^{(0)} & =p_{x}(0) \\
u^{(0)} & --p_{y}(0) \\
p_{z}(0) & =-S^{(0)} \\
u_{x}(0)+v_{y}(0) & =0 \\
S_{z z^{(0)}} & =0
\end{aligned}
$$

In analyzing the Ekman layers, one must stretch the vertical coordinate according to $\bar{z}=\varepsilon_{v}^{-1 / 2} z$ or $\bar{z}=\varepsilon_{v}^{-1 / 2}(z+d)$ for top and bottom Ekman layers respectively. Then, always in the context of expansion [7], the Ekman zero order system is:

$$
\begin{aligned}
-v^{(0)} & =-p x^{(0)}+u_{\bar{z} \bar{z}}(0) \\
u^{(0)} & =-p_{y}(0)+v_{\bar{z} \bar{z}}(0) \\
p_{\bar{z}}^{(0)} & =0 \\
u_{x}(0)+v_{y}^{(0)}+w_{\bar{z}}^{(0)} & =0 \\
S_{\bar{z} \bar{z}}^{(0)} & =0
\end{aligned}
$$


Following the usual procedure of splitting the velocity components into an interior (geostrophic) part plus an Ekman layer contribution which must decay outside the layer itself, it can esaily be found that the Iskman velocities are:

$$
\begin{aligned}
& u_{E^{(0)}}=e^{\bar{z} / \sqrt{2}}\left(b \sin \left(\frac{\bar{z}}{\sqrt{2}}\right)+c \cos \left(\frac{\bar{z}}{\sqrt{2}}\right)\right) \\
& v_{E}^{(0)}=e^{z / \gamma^{\prime} \overline{2}}\left(c \sin \left(\frac{\bar{z}}{\sqrt{2}}\right)-b \cos \left(\frac{\bar{z}}{(\sqrt{2})}\right)\right.
\end{aligned}
$$

The integration constants $b$ and $c$ are determined applying the bomdary conditions which, for the top and bottom layers, are respectively:

$$
\begin{aligned}
& \partial u_{t o t}(0) / \partial z=\partial\left(u_{\text {interior }}{ }^{(0)}+u_{E}^{(0)}\right) / \partial z=\tau_{w^{x}} \\
& \partial v_{t o t}(0) / \partial z=\partial\left(v_{\text {interior }}{ }^{(0)}+v_{E^{(0)}}\right) / \partial z=\tau_{w^{y}}
\end{aligned} \text {, at } z=0
$$

where $\tau_{\mathbb{W}^{x}}, \tau_{\mathbb{W}^{y}}$ are the dimensionless wind stress components at sea surface $z=0$, and

$$
u_{t o t}^{(0)}=u_{\text {interior }}{ }^{(0)}+u_{E}^{(0)}=0 ; \quad v_{t o t^{(0)}}=v_{\text {interior }}{ }^{(0)}+v_{L^{(0)}}=0
$$

at $z=-\lambda$, no slip condition at the sea bottom. The vertical velocity component $w_{t o t}^{(0)}=w_{\text {interior }}^{(0)}+w_{E}^{(0)}$ can be easily found from the continuity equation and top and bottom boundary conditions, in our case $w_{t o t a} t^{(0)}=0$ at $z=0$, - $d$. The zero order velocity field is therefore completely determined. The most important point to be noticer, anyway, is that the Ekman layers are boundary layers for what momentum is concerned but not for the density and pressure fields. Apart from the physical consideration that the Ekman layer is characteristically the one in which rertical diffusion of momentum is the dominant process, this can be rigorously shown considering the Ekman layer form of the density and pressure equations. From these, as any Ekman correction to the interior fields must decay ontside the layer's themselves, it is evident that these corrections must be idlentically zero. Therefore, the interior pressure and density determine the total fields of these quantities.

From the zero order equation $S_{z}(0)=0$,

$$
S(0)=A(x, y, t) z+B(x, y, t)
$$


The proper surface and bottom boundary conditions for the density are:

$$
I_{z} S_{z}=Q \quad \text { at } z=0 ; \quad S_{z}=0 \quad \text { at } z=-d
$$

At $z=0$, the air-sea interface, the source function $Q$ includes all heat and water vapor fluxes which produce density changes through cooling and eraporation. $\Delta \mathrm{t} z=-\boldsymbol{d}$, no density flux is recluired through the sea bottom. In terms of the expansion [7], this means:

$$
\begin{gathered}
\Gamma_{v} S_{z}^{(0)}+\Gamma_{v} \Gamma_{I I}^{a} S_{z}^{(1)}+\ldots=\Gamma_{v} S_{z}^{(0)}+\gamma S_{z}^{(1)}+0\left(I_{H}^{a}\right)=0 \\
\text { at } z=0 \quad ; \quad S_{z}^{(0)}=S_{z}(i)=\ldots=0 \quad \text { at } z=-l
\end{gathered}
$$

From the bottom boundary condition, it follows:

$$
S^{(0)}=S^{(0)}(x, y, t)
$$

The zero order density is not vertically dependent. From [17] and the surface boundary condition, it follows that the proper choice for the latter is:

$$
\gamma \tilde{s}_{z^{(i)}}=Q \quad \text { at } z=0
$$

It is therefore rigorously shown that, under the set of assumptions defining our physical system, the zero order density depends only on horizontal coordinates. The zero order pressure equation can hence be integrated to give:

$$
p^{(0)}=\pi(x, y, t)-S^{(0)}(x, y, t) \cdot z
$$

where $\pi(x, y, t)$ is a surface pressure fielt.

The $\Gamma_{I I}^{a}$ order equation for the density can be written as:

$$
S_{t}^{(0)}+J\left(\psi^{(0)}, S^{(0)}\right)=\gamma S_{z z^{(1)}}
$$

where $u^{(0)}=-\psi_{\mu^{(0)}}: v^{(0)}=+\psi_{x^{(0)}}$ define a zero-order stream function through the continuity equation, and $J(a, b)=\partial a / \partial x \times \partial b / \partial y-$ $\partial a / \partial y \times \partial b / \partial x$ is the Jacobian of the fields $a, b$.

If $J\left(\psi^{(0)}, S^{(0)}\right)=0$ - which implies $\psi^{(0)}=f\left(S^{(0)}\right)$ - and if the source function $Q$ is only a function of time, the density equation [20], integrated from top to bottom, admits the exact solution:

$$
S^{(0)}=S_{i n}(0)+\frac{1}{d} \int_{1}^{b} Q\left(t^{\prime}\right) d t^{\prime}
$$

and $S_{i n}{ }^{(0)}$ is the density field at $t=0$. 
The momentum equations previously discusser, if integrater from top to bottom and properly treated in terms of a transport stream function, produce, together with the density equation [20] also integrated vertically, the morlel equations of the previously mentioned relerence, of which equation [21] was a particular solution, confirmed by comparison with the experimental data.

The only diflerence is that the density morlel equation of Hendershott-Rizzoli maintained a horizontal difiusion term $\Gamma_{H} \nabla_{H}{ }^{2} S$, which, in the treatment through the expansion procerlure [ 7 ], will appear only at higher orlers. We must point out, anyway, that the analysis here made is rigorously valid only in the interior of the basin, away from sirle wall boundaries, where horizontal density gradients, and therefore horizontal diffusion terms, become much more important, and can be inferred to be not negligible. Near these side walls, this expansion procedure in terms of the small parameter $\Gamma_{H}$ ought to be inserted in the context of a boundary layer analysis, emphasizing the importance of the diffusion processes responsible for the mixing of the coastal fresh water with the denser one of the interior, and therefore bringing to zero order the horizontal diffusive term.

In the mathematical morlel previously mentioned, no distinction was made between the interior and the side wall boundary, the morlel describing the evolution of circulation and density fields in the whole of the basin. The diffusive term, even weighted by $\Gamma_{H}$, was therefore necessary to allow for the mixing of coastal and interior waters, and appeared to be of fundamental importance in determining the time evolution of the properties of the basin.

Even with these points in mind, the preceding analysis shows that, for a sea like the Arliatic and under the physical assumptions apt to morlel a Winter characteristic situation, the main interior part away from a sicle wall coastal strip obeys the set of equations [15], learling to the detailed solutions previously ontlined.

\section{5) Comparison Wi'tl EXPERIMENTAL RESLLTS}

Having rigorously justified the basic assumption $S=S(x, y)$ of the morlel, numerical experiments have been carriel out; the results of a typical computation are shown in Fig. 3 and 4.

In them, the evolution of the transport stream function $\psi$ and density field $S$ are shown at successive steps of the time integration. 

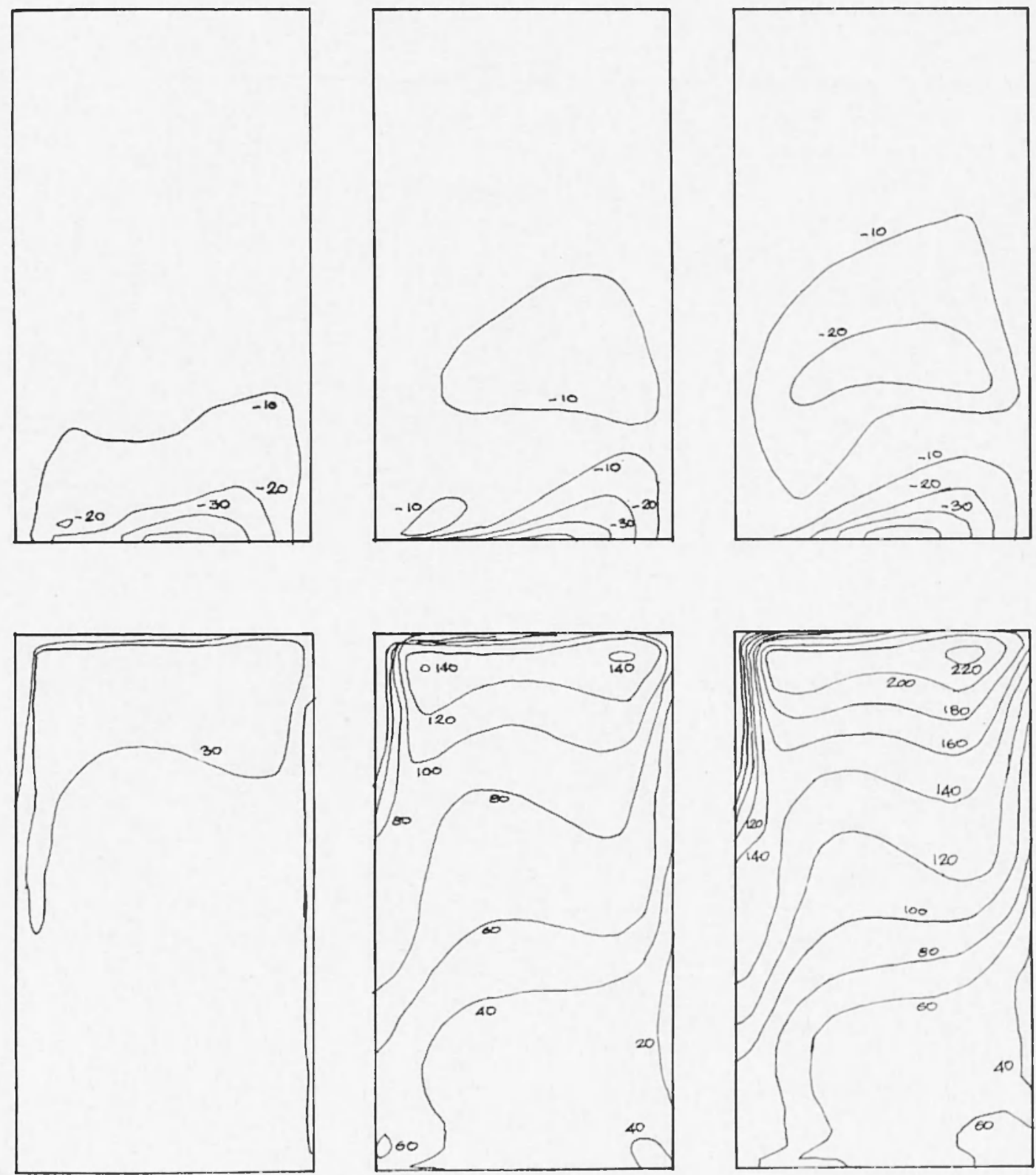

Fig. 3 - Time evolution of transport stream function (a1-a2-a3) and density (b1-b2-b3) both in dimensionless units, multiplied through 100 .

Streau function unit: $10^{8} \mathrm{~m}^{3} / \mathrm{sec}$. lensity units: $=29.0+\Delta \sigma \mathrm{S}$ with $=0.5$ a $1-b 1=$ time step 5, corresponding to 3.46 days - a 2 - b2 = time step 15, corresponding to 10.38 days - a3-b3= time step 25 , corresponding to 17.38 days 

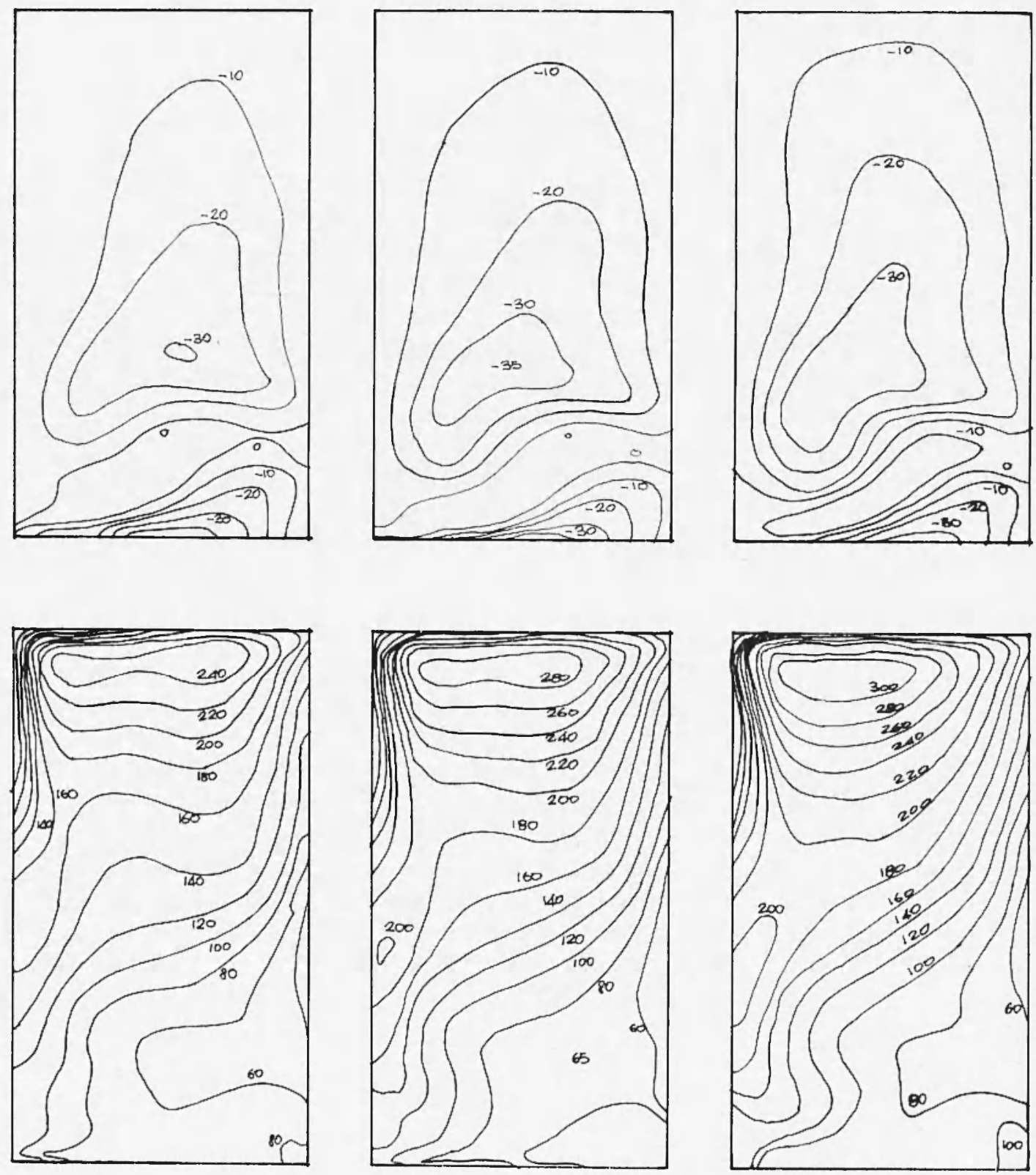

Fig. 4 - As in Fig. (3), transport stream function (a1-a2-a3) and density (b1-b2-b3).

al - bl $=$ time step 35 , corresponding to 24.22 days

$\mathrm{a} 2-\mathrm{b} 2=$ time step 45 , corresponding to 31.14 days

$\mathrm{a} 3-\mathrm{b} 3=$ time step 55 , corresponding to 38.10 days 


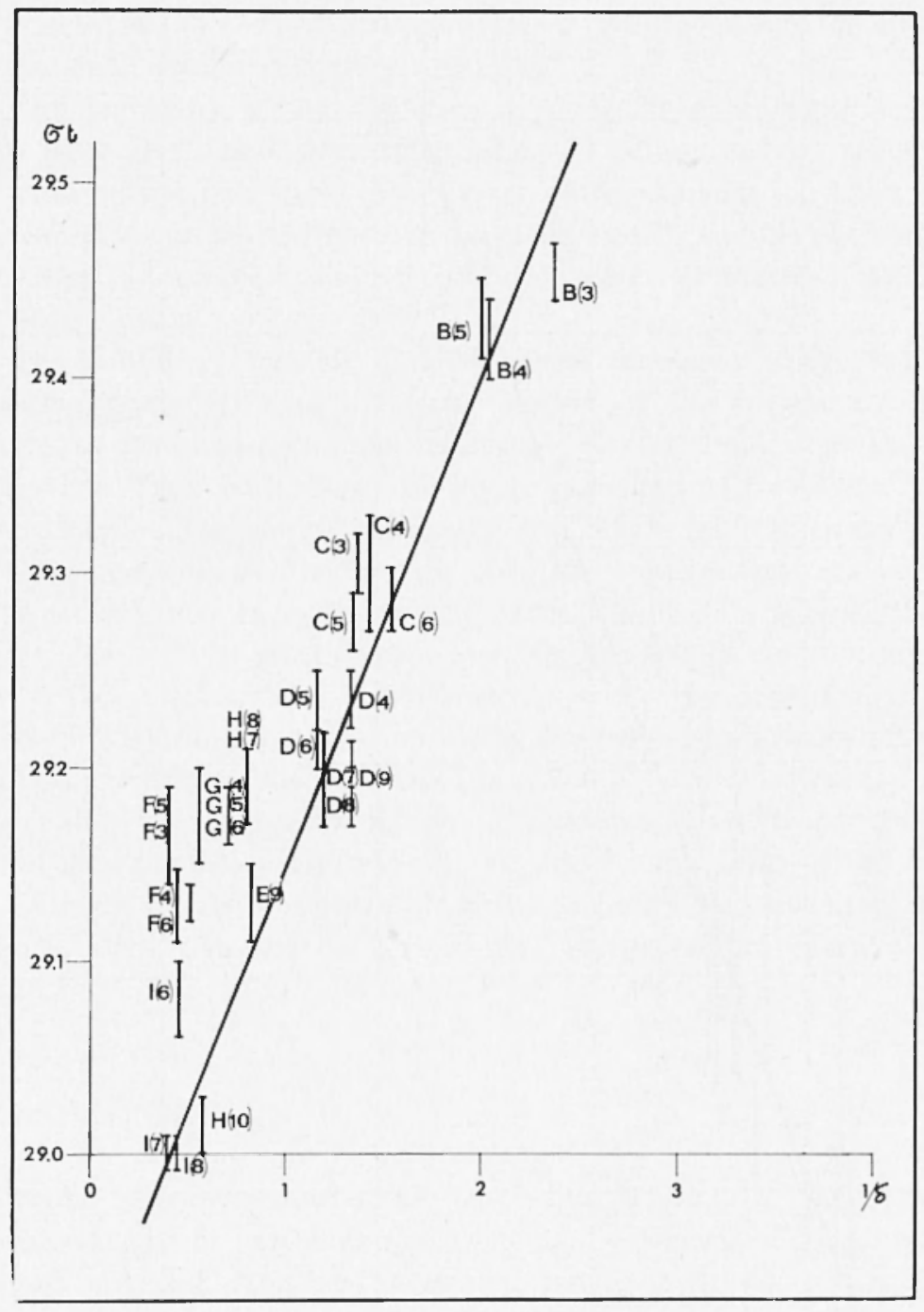

(a)

Fig. 5 a-b $-\sigma_{\ell}$ as plotted versus dimensionless inverse depth for the cruise of Winter 1966 (a) and Winter 1972 (b). 


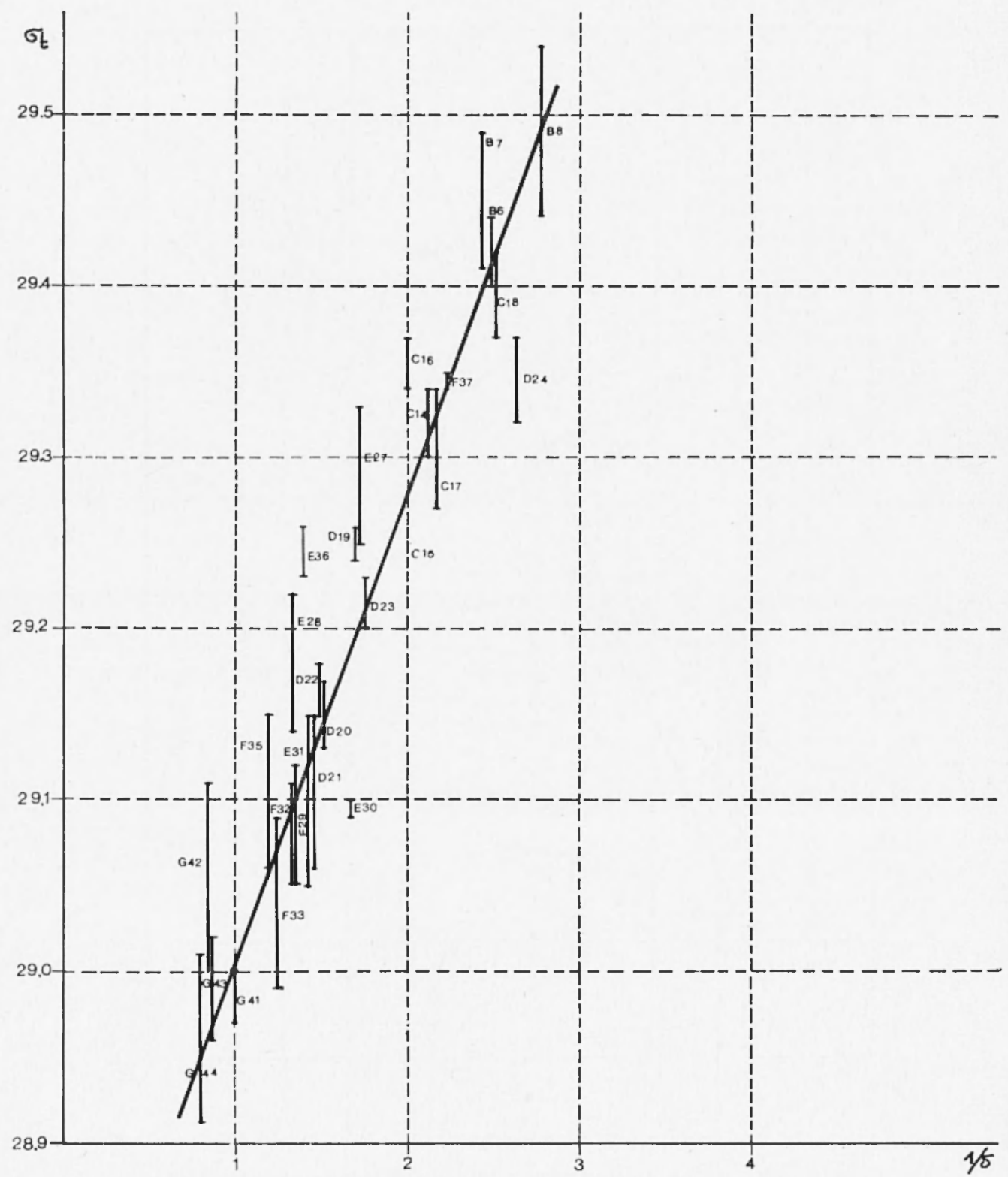

(1) 
It is evirlent the formation of the rlense water pool centered in the Northern part of the basin, stretching sonthwarl in the characteristic tail which follows the isobath contours, in rery good agreement with experimental results (compare with Fig. 2).

The transport stream function $\psi$ shows a characteristic circulation gyre of thermohaline origin, that is determined by the strong grarlients between the light water river outflows along the boundaries and the rlense water of the interior. This grre is completely indipendent from the forcing boundary condition given at the open southern end of the basin.

The analytical solution [21] has been, moreover, compared vitl the experimental data at disposal. In Fig. 5 the results are given relative to the oceanographic cruises of Winter 1966 and 1972 respectively. They both show the linear dependance of $\sigma$, from the inverse dimensionless depth $1 / d=D_{\mathrm{o}} / D=100 \mathrm{~m} . / D$. Excluded from the linear rlepenrlance region are only the undercoastal stations, in the Western strip arljacent to the Italian shoreline, where, also in Winter, the vertical stratification persists because of river water outflows. This means that, in this coastal strip, the rependance from the vertical coordinate $z$ must appear in the zero order density function $S^{(0)}$, and not only in the first order function $S^{(1)}$ as it has been proved to be valid for the interior region. Therefore, this strip can be consirlerer as a boundary layer in relationship to the whole of the basin, and as such, it can be treater with the techniques of boundary layer analysis, always in the context of the expansion procerlure refiner by $[7]$.

\section{6) Conclusions}

Under the definition of a penetration depth, we have pointed out the variety of mathematical systems - corresponding to widely dilferent physical situations - which can arise in the context of a rigorous formal procedure. We have therefore applied this procerture to the specific case of a Winter hydrologic situation in the Arlriatic Sea, showing how the rensity field can be assumed, to a zero order approximation, to be vertically homogeneous, and re-deriving a set of solutions morlelled in a previous paper.

For this sturly to be complete, a boundary layer analysis must be arded to take into account the modifications of the model equa- 
tions describing the interior fields, when approaching a side wall coastal boundary. In it, horizontal diffusion processes - of higher order in the interior - will become important in producing the local dynamics of mixing and in influencing as a consequence, the evolution itself of the interior lields.

Studies in this direction have been undertaken, and considerable progress is being made.

TABLE OF SYHBOLS

$A_{v}=$ vertical eddy viscosity coefficient

$A_{H}$ - horizontal eddy viscosity coefficient

$K_{v}=$ vertical eddy diffusivity coefficient

$\hbar_{H}=$ horizontal eddy diffusivity coefficient

$X \quad=$ horizontal length seale

$D_{o}=$ vertical length scale

$f=$ Coriolis parameter

$\varrho_{o} \quad=$ mean density of the basin

$U=$ velocity scale

$\Delta \sigma=$ scale for horizontal variations of density anomaly $\sigma_{\ell}$

$D_{e}=\sqrt{A_{v} / /_{o}}$ Ekman depth

$T_{a d v}=\mathrm{X} / \mathrm{U}$ advective time seale

$T_{v d}=D_{v}-/ K_{v}$ vertical diffusion time seale

$T_{H d}=\mathrm{X}^{2} / K_{H}$ horizontal diffusion time scale

$V_{H^{2}}=\partial^{2} / \partial r^{2}+\partial^{2} / \partial y^{2}$ horizontal Laplacian

$\varepsilon_{T}=1 / \int_{0} T$

$\varepsilon_{R}-U / /_{o} \mathrm{X}$ Rossby number

$\varepsilon_{v}=A_{v} / f_{o} D_{o}{ }^{2}$ vertical Ekman number

$D_{s}{ }^{2}=K_{v} X / U$ penetration depth

$\Gamma_{v}=T_{a d v} / T_{v d}=D_{s}-1 D_{o}^{2}$

$\Gamma_{H}=T_{a d v} / T_{H d}=K_{H} / \mathrm{X} \cdot U$

$\varepsilon_{H}=A_{H} / f_{o} X^{2}$ horizontal Ekman number

$d \quad=D / D_{o}$ dimensionless depth 


\section{REFERENCES}

(1) IInNdenshott, M. C. - Rizzoli, P. 1976. - The Winter Circulalion of the Adriatic Sea. "Deep Sea Research", 23, n. 5, pp. 353.

$\left({ }^{2}\right)$ Malaxotte Rizzol. P. 1977. - Winler Oceanographic properlies of Northern Adrialic Sea. Cruise Jamuary-February 19\%2. In press on Archivio di Oceanografia e Ijimnologia.

(3) Pentosky, J. 1974. - On coastal Jels and Cpuelling in Bounded Basins. "Journ. of Physical Oceanography", 4, pp. 3.

(4) Proloskr, ,1. 1974. - Longshore Currents, Upwelling and Botlom Topoqraphy. "Journ. of Physical Occanography", 4, pp. 214.

(5) PEDLoskY, J. 1974. - Longshore Currents and the Onsel of Cpucelling oner Botlom slope. "Journ. of Physical Oceanography", 4, pp. 310.

(6) Trotт', 1. 1970. - Crociere Mare Adrialico 1965-66. "Servizio Nazionale Raccolta Dati Oceanografiei", Serie A. Commissione Italiana per loceanografia, C.N.R.

Questo lavoro ¿ stato eseguito nell ambito del Progetio Finalizzato Oceanografia. 